\title{
COMENTARIOS SOBRE SWEEZY Y SU INTERPRETACION EN LUSTIG
}

\author{
Firdans Jhabuala \\ Instituto Tecnológico Regional de Oaxaca
}

\section{Lo que Sweezy escribe y Lustig entiende}

LA GRAN DEPREsión de los años treinta.suscitó el interés de los economistas por explicarla: Las explicaciones de este fenómeno que provocaron mayor impacto han sido las de Kalecki, Keynes, Sweezy y Steindl. ${ }^{1}$ A diferencia de los trabajos de Kalecki y Keynes, el trabajo de Sweezy fue publicado después del inicio de la Segunda Guerra Mundial, es decir, luego que la economía capitalista mundial superara la Gran Depresión y posiblemente por esta razón no recibió la merecida atención.

El capitalismo mundial se recuperó de la Gran Depresión con el inicio de los preparativos para la Segunda Guerra Mundial y no hubo ninguna crisis de importancia hasta mediados de los años sesenta cuando comienza un ciclo de crisis reiteradas. La renovación del estado permanente de crisis en el centro del sistema mundial capitalista ha despertado un nuevo interés tanto por el trabajo de Sweezy como por el de Steindl. Este ha sido acompañado por renovados malos entendidos y malas interpretaciones del famoso texto de Sweezy y un buen ejemplo de estos es el artículo de Nora Lustig ${ }^{2}$ publicado en "Demografía y Economía" de El Colegio de México.

1 Véase en particular, Michal Kalecki, The Theory of Economic Dynamics, Monthly Review Press, New York, 1968; John Maynard Keynes, "The General Theroy of Employment, Interest and Money", Harcourt, Brace and World, Inc., New York, 1935;

Paul Sweezy, The Theory of Capitalist Development, Monthly Review Press, New York, Third Printing of First Modern Reader Paperback Edition, 1968; y Joseph Steindl, "Maturity and Stagnation in American Capitalism", Monthly Review Press, New York, 1976.

2 Lustig, Nora, "Una Crítica al Modelo Subconsumista de Paul Sweezy y Perspectivas para una Reformulación", "Demografía y Economía, Vol. XIII, No. 2, 1979 pp. 155-167. 
Lustig intenta demostrar los supuestos errores de Paul Sweezy, 37 años después de la primera publicación de su libro, en un momento en que las recientes crisis del capitalismo han facilitado la comprensión del trabajo y empieza presentando esquemáticamente lo que cree son las "dos grandes corrientes" dentro "de las teorías marxistas sobre la crisis del sistema capitalista" 3 . Califica a Sweezy de uno "de los más vehementes propulsores de las hipótesis subconsumistas como explicación de la crisis del capitalismo, sobre todo del capitalismo maduro"3, y se adelanta a reproducir extractos de un par de páginas del libro de Sweezy.

Como lector de las diferentes obras de Sweezy y sus editoriales en Monthly Review, me pareció raro que alguna vez Sweezy pudiera haber sido uno de los más vehementes propulsores mencionados por Lustig $^{4}$. Inmediatamente, me referí al índice general del libro en cuestión, en el cual la tercera parte (a la cual se refiere Lustig), está dedicada a las crisis y las depresiones y consta de los Capítulos VIII a XII, en los cuales solamente la segunda sección del Capítulo X, su Apéndice (a lo cual regresaremos más adelante) y las secciones 3 y 4 del Capítulo XII, están explícitamente dedicadas al papel del subconsumo en las crisis y las depresiones. ${ }^{5}$

Dentro de este esquema general ¿de dónde sacó Lustig la noción de que Sweezy es uno de los más vehementés propulsores de las hipótesis subconsumistas? Si así fuera, ¿no sería razonable esperar que Sweezy dedicara más de una sección de un Capítulo de una parte de uno de sus libros al tema? En ninguna parte de las páginas del libro de Sweezy se percibe la idea de que Sweezy se limita al subcon-

3 "Lustig", p. 155.

4 Es posible que Lustig confiara demasiado en la opinión de críticos del subconsumismo en general, como es el caso de Michael Bleaney, Teoría de las Crisis, Editorial Nuestro Tiempo, México, D. F., 1977, especialmente pp. 142-148.

Sin embargo, el punto esencial de Bleaney en estas páginas es que Marx no se categoriza como subconsumista a pesar de las referencias aducidas por Sweezy en su texto. Más adelante, en el Capítulo XII Bleaney regresa al problema de la deficiencia de la demanda agregada crónica en el capitalismo maduro, un tema del libro de Steindl desarrollado por Sweezy (junto con Paul Baranj en el cual el subconsumo del sistema es una parte importante del problema de la realización de plusvalía. Ciertamente, el tema central del libro no es el subconsumismo sino la inhabilidad creciente del capital monopolista para realizar la plusvalía ya creada y encarnada en la abundante producción relativa a la demanda agregada de la economía, y una buena parte del libro trata de los medios del capital monopolista para resolver o reducir esta contradicción fundamental. Ahora bien, en qué grado esas hipótesis son keynesianas o marxistas, es otra pregunta fuera de mi tarea inmediata.

5 Sweezy, Paul M., Teoría del Desarrollo Capitalista, Fondo de Cultura Económica, Cuarta Reimpresión de la Primera Edición en Español, 1970, p. 429. 
sumo para explicar las crisis y las depresiones del capitalismo. Esta impresión es aún más clara cuando uno lee las líneas que inmediatamente siguen los extractos de las dos páginas reproducidas en el artículo de Lustig, y que aquí reproduzco: ${ }^{6}$

"Hemos hablado de una tendencia del consumo a quedarse atrás de la producción total de artículos de consumo. Sin embargo, puesto que el numerador y el denominador, en las dos precedentes proporciones, están funcionalmente relacionados de tal forma que sería imposible sustraer de uno sin sumar al otro, es igualmente lógico hablar de una tendencia del abastecimiento de medios de producción a exceder las necesidades de medios de producción. Bien entendidos, por lo tanto, el 'subconsumo' y la 'sobreproducción' son las dos caras de una misma moneda. Si se tiene presente esto, no será motivo de sorpresa el que una crisis de 'subconsumo' pueda estallar primero en la esfera de la producción de medios de producción, mientras que una crisis de 'sobreproducción' puede estallar primero en la esfera de la producción de artículos de consumo. La etiqueta que se use es una cuestión de gusto, y el punto de origen un detalle relativamente sin importancia, que depende de una multitud de circunstancias particulares.

"Debe señalarse de nuevo que aquí tenemos que ver con una tendencia al subconsumo, que siempre está presente pero que puede ser total o parcialmente contrarrestada por fuerzas contrarrestantes que no han sido tomadas en cuenta todavía. De la naturaleza de tales fuerzas contrarrestantes y de su vigor relativo en diferentes etapas del desarrollo capitalista, trataremos en el capítulo XII."

Como se puede observar, para Sweezy el subconsumo es un aspecto importante pero al mismo tiempo, elemento componente de la problemática de la depresión y la crisis. Así " es "de esperarse que la exposición hecha en este capítulo sirva para eliminar las dudas y vacilaciones que han impedido hasta ahora a muchos economistas marxistas aceptar la teoría del subconsumo como un aspecto - y un aspecto muy importante- de todo el problema de la crisis."

Como hemos visto, las ideas de Sweezy no se reducen a unas recetas o fórmulas subconsumistas y tampoco al Apéndice Matemático del capítulo X, pero Lustig acentúa la importancia del Apéndice y sustenta que "en dicho modelo se encontraron una serie de inconsistencias que invalidan su adjudicada función sistematizadora". ${ }^{8}$

6 ibid., pp. 203-204.

7 ibid., p. 207.

8 Lustig, p. 157. 
Nosotros creemos, por el contrario, como veremos más delante, que los cálculos de Lustig muestran una serie de inconsistencias que invalidan su función crítica. Antes de llegar a un examen del Apéndice y su crítica por parte de Lustig, queremos dejar en claro:

1) Las tendencias que trabajan en contra del estancamiento que Sweezy trata en el Capítulo XII no han sido siquiera mencionadas por Lustig. ${ }^{9}$

2) La crisis actual del centro del capitalismo, fue pronosticada por Sweezy en estas líneas no consideradas por Lustig: ${ }^{10}$

"Es muy posible que el ingreso nacional aumente a una tasa ascendente en un país capitalista 'joven', en el que la fuerza humana abunda o aumenta con rapidez. Nuestro análisis sugiere, por lo tanto, que es improbable que tal país tenga que afrontar serias dificultades de subconsumo. Pero en un país capitalista 'viejo' - y todos los países capitalistas avanzados con la posible excepción del Japón merecen sin duda, esta designación hoy día- es casi seguro que el ingreso nacional aumenta a una tasa descendente. En lo que concierne al capitalismo tenemos indudablemente razón en llamar al subconsumo una enfermedad de la vejez. Para argumentos adicionales en apoyo de esta conclusión, referimos al lector al capítulo XII."

No creo posible tratar la teoría marxista de la crisis en unas páginas, y mi intención hasta este momento no ha sido ésa, sino comprobar la pobre interpretación dada por Lustig a las ideas de Sweezy. Por si esto no fuera suficiente, Lustig se interna en un análisis matemático del Apéndice al capítulo X del libro de Sweezy.

Repetiremos brevemente la formulación de Sweezy en este Apéndice: $\mathrm{El}$ ingreso nacional neto, I, se divide en tres partes: $\mathrm{s}$, los salarios de los trabajadores; $e$, la parte de plusvalía consumida por los capitalistas; y $\mathrm{k}$ la parte de plusvalía invertida por los capitalistas. Ahora bien, los últimos dos conceptos crecen más lentamente en términos absolutos $y$ relativos para cualquier nivel de $k$ que el crecimiento de $\mathrm{k}$ mismo, un punto no comprendido por Lustig como evidencia su nota de pie de página No. 8. Se puede argumentar

9 El desarrollo posterior de este tema por Sweezy con Paul Baran, resultó en el libro "El Capital Monopolista" que analiza el papel del Estado en la absorción de plusvalía como una de las tres tendencias principales hacia la absorción de plusvalía en las economías capitalistas desarrolladas. La crisis fiscal que resulta es tema de James O' Connor, "The Fiscal Crisis of The State" St. Martin's Press, New York, N. Y., 1973.

10 Sweezy, “Teoría....", pp. 209-210. 
en términos Keynesianos que s y $e$ son funciones de consumo y crecen más lentamente que el ingreso (I) y por implicación la plusvalía (k),o se puede argumentar que la meta principal del capitalista es acumular y su consumo figura solamente como meta secundaria. Por otra parte, la disminución relativa de los ingresos de los trabajadores (y como consecuencia su consumo) frente a la acumulación de los capitalistas se debe a la naturaleza del sistema de propiedad privada. ${ }^{11}$ Sweezy llama "un rasgo fundamental del capitalismo el que una proporción creciente de la plusvalía tiende a ser acumulada y una proporción creciente de la acumulación tiende a ser invertida, tanto $s$ como $e$ deben aumentar menos rápidamente que $\mathrm{k}^{.12}$

Ahora bien, es posible que el consumo no esté relacionado con la plusvalía de esta manera, sin embargo, si queremos comprobar la inconsistencia interna del modelo, estamos obligados a aceptar la hipótesis planteada. En la parte 7 de la sección 4 de este artículo, ampliaremos la información sobre este tema. Aquí anotamos que las funciones resultantes:

$$
\mathrm{s}=\mathrm{f}(\mathrm{k}), \quad \quad e=\mathrm{g}(\mathrm{k})
$$

tienen primeras derivadas entre 0 y 1 y segundas derivadas que son negativas para toda $\mathrm{k}$ positiva y finita, según el Apéndice de Sweezy.

Una función $\mathrm{f}(\mathrm{k})$ se considera cóncava si existe un escalar $\alpha, 0 \leqslant$ $\alpha \leqslant 1$, tal que $\mathrm{f}\left[\alpha \mathrm{x}_{1}+(1-\alpha) \mathrm{x}_{2}\right] \geqslant \alpha \mathrm{f}\left(\mathrm{x}_{1}\right)+(1-\alpha) \mathrm{f}\left(\mathrm{x}_{2}\right)$ para cualquier . $\left(\mathrm{x}_{1}, \mathrm{x}_{2}\right) \in \mathrm{x},{ }^{13}$ es decir, si la línea que conecta dos puntos en la cur-

$11 \mathrm{La}$ disminución es absoluta en el caso de las viejas economías capitalistas, especialmente los imperialismos en fase de declinación como el inglés y el norteamericano. Por ejemplo, en la actualidad el "take-home pay" del trabajador norteamericano con tres dependientes es casi el mismo en términos reales que en 1965 y en los últimos tres meses (Dic. 1979-Feb. 1980) ha sufrido disminuciones del $0.4 \%, 1.3 \%$ y $1.4 \%$ (respectivamente). (Véase periódico El Excélsior de México del 26 de marzo de 1980 , p. 26-A).

12 Sweezy, "Teoría. ...", p. 208.

13 Véase Blaine Roberts y David L. Schulze, Modern Mathematics and Economic Analysis, W. W. Norton \& Co., Inc., 1973, p. 374. Mientras que los economistas y algunos matemáticos dan esta definición de la función cóncava, la literatura matemática está llena de términos como "cóncava hacia arriba" y "cóncava hacia abajo", que son lơs términos de Louis Leithold, "El Cálculo", HARLA, segunda edición, México, 1973, pp. 227, 228. Incluso, los matemáticos llaman una curva cóncava cuando otros la llamarían "convexa" que es el caso en Elementos de Matemáticas Superiores de I. L. Zaitsev, MIR, Moscú, 1977, pp. 235-237; y en "Nonlinear Programming" de H. W. Kuhn y A. W. Tucker en "Proceedings of the Second Berkeley Symposium on Mathematical Statistics and Probability", ed. Jerzy Neyman, Berkeley, 1950, p. 481. Para un resumen de los términos, véase Tjalling Koopmans "Three Essays on the State of Economic Science", McGraw Hill Book Co., Inc., New York, 1957, p. 27 y A. A. Walters, "An Introduction to Econometrics", W. W. Norton \& Co. Inc., New York, 1970 , p. 273 . Hemos usado el término "cóncava" en este sentido que es el que predomina en la literatura de la economía. 
va de $f(x)$ está siempre abajo de la curva, entonces $f(x)$ es cóncava; según la definición, $f(k)$ y $g(k)$ son funciones cóncavas. Por lo tanto, $\mathrm{I}(\mathrm{k})$ también es cóncava. ${ }^{14}$ Ahora bien, solamente de las condiciones (1)-(3) en Lustig $^{15}$ sobre $I(k)$ se puede asegurar que $\ddot{\mathrm{I}}<\ddot{\mathrm{k}}$ cuando el primero de estos no es positivo. ${ }^{16}$ Sweezy tomó otra línea para llegar al mismo punto y hace la demanda para nuevas máquinas, $\mathrm{c}$, depender de una manera fija sobre el aumento de consumo. Por simplificación, definiremos $\mathrm{x} \equiv \mathrm{s}+e$, el consumo total dé la economía.

Así, $c=\lambda \dot{x}$ donde $\lambda$ es el acelerador y c es el aumento a los medios de producción para lograr el aumento del consumo dx en un tiempo dt.

Ahora bien, si $\stackrel{\circ}{\mathrm{c}} \neq \stackrel{\circ}{\mathrm{k}}$, habrá una contradicción entre las tasas de aumento de capital como nueva maquinaria y como plusvalía dictado por:

1) Los requerimientos del consumo (de trabajadores y capitalistas); $\mathrm{y}$

2) Los requerimientos de acumulación de plusvalía (de capitalista).

Derivando $\mathrm{c}$ con respecto a tiempo y sustituyendo la identidad de $\mathrm{I} \equiv \mathrm{s}+e+\mathrm{k}$ obtenemos

$$
\stackrel{\circ}{c}=(\ddot{I}-\ddot{k})
$$

14 Roberts y Schulze, p. 424.

15 Lustig, p. 158.

16 Cada punto representa una derivada con respecto a t. Así,

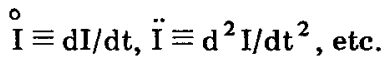

Ahora, I es una función de $\mathrm{k}$ y por lo tanto,

$\ddot{\mathrm{I}}=\mathbf{a}+\mathbf{b} \ddot{\mathbf{k}}$, donde

$\mathrm{a} \equiv \mathrm{I}^{\prime \prime}(\mathrm{k}) \stackrel{\circ}{\mathrm{k}}^{2} ; \mathrm{b} \equiv \mathrm{I}^{\prime}(\mathrm{k}), 1<\mathrm{b}<3$.

Veamos que a $<0<$ b y como consecuencia una gráfica de la línea que relaciona $\ddot{k}$ a $\ddot{I}$ para cualquier $(t, k, \dot{k})$ determinadas muestra que $\ddot{k}>\ddot{I}$ si es último no es positivo. Se presenta un desarrollo completo de esta propiedad más adelante con el apoyo del Diagrama 3 en la Sección 3; sin embargo, por la concavidad de $I(k)$ se puede concluir que $k>I$ cuando este último no es positivo. 
que será negativa si $(\ddot{\mathrm{I}}-\ddot{\mathrm{k}})<0$.

Por otro lado, $I=f(k)+g(k)+k$.

$\therefore \quad \ddot{I}=\left(f^{\prime}+g^{\prime}+1\right) \ddot{k}+\left(f^{\prime \prime}+g^{\prime \prime}\right)(\dot{k})^{2}$.

Aquí, en su ecuación (8), Lustig hace un claro error de derivación por ignorar que para derivar con respecto a t hay que derivar antes con respecto a $\mathrm{k}$ que nos dejará con $(\mathrm{k})^{2}$ y no $\mathrm{k}$ en el segundo término del lado derecho de la ecuación diferencial. ${ }^{17}$

Sweezy explícitamente excluye el caso de las economías jóvenes ${ }^{18}$ que implica un ingreso nacional creciente en forma acelerada $(\dot{I}>0) .{ }^{19}$ Para las economías que no exhiben esta característica, Sweezy concluye que $\ddot{\mathrm{k}}>\ddot{\mathrm{I}}$ por la cuál $\dot{\mathrm{c}}<0$ cuando $\ddot{\mathrm{k}}<0$. Esto no parece razonable a Lustig y vale la pena repetir sus argumentos.

\section{Las leyes del cálculo y las leyes del capitalismo.}

Lustig escribe: "En la sección del libro que dedica a ese tema, Sweezy menciona la necesidad de hacer una formulación cuidadosa de esta

17 Esto no puede ser un error de impresión como podía ser la confusión entre " $e$ " $e$ " $I$ " en las ecuaciones $(5)$ y (6) de Lustig, p. 159, porque ella argumenta, basándose en Sweezy, que $k>0$ (Lustig, p. 161).

18 "Teoría. ...", p. 209.

19 Lustig no entiende en su nota de pie \# 7 que salvo el caso de una economía capitalista cuyo ingreso nacional crece en forma acelerada, las otras economías capitalistas tienden a estacionarse. La tendencia puede resultar más débil que otras durante algún tiempo, pero se reivindicará en el largo plazo. Así la tendencia para la tasa de ganancia a disminuir puede ser revertida por el descubrimiento de un país que no ha sido saqueado. El botín inesperado aumentará la tasa de ganancia durante el período del saqueo; sin embargo, para rendir estas tasas altas se requiere descubrir otro país no saqueado que rinda un botín mayor que el primer pais en la proporción de la ganancia invertida del primer botín al capital inicial arriesgado en la aventura. Por otra parte, la presencia de una tendencia no necesita ser palpada para que exista. Considérese un coche que cuenta con poca gasolina y cuyo chofer lo está acelerando; aunque el coche se está acelerando, tenderá a pararse unos momentos después. Es decir, el comportamiento actual y momentáneo puede ser contrario a la tendencia. En este sentido siempre existe la tendencia hacia el estancamiento de la plusvalía y el ingreso nacional. 
teoría: ${ }^{20}$ con este fin, introduce un modelo algebraico en el apéndice que $\rightarrow$ según Sweezy - sirve como sustentación lógica de tal formulación". ${ }^{21}$

Lustig recurre ahora a la brujería algebraica para quitar los malos espíritus del cuerpo enfermo del capitalismo. Se le ocurre que İ puede ser $\gtrless 0$ y sobre esta base construye "tres posibles alternativas". ${ }^{22}$ Vamos a examinarlas:

a) Cuando $\ddot{\mathrm{I}}<0$, Lustig concluye que $\ddot{\mathrm{k}}$ "puede ser mayor, igual o menor que $d^{2} I / d t^{2}$, según sean las magnitudes de los demás componentes, las cuales constituyen deseconomía que los supuestos del modelo no permiten inferir". 22 Pero esto no es cierto, ya que sí es posible inferir que $\ddot{k}>\ddot{I}$ como hacemos en la próxima sección. Lustig no llega a esta conclusión por no ver el problema más detenidamente.

b) Cuando $\ddot{\mathrm{I}}=0$. Aquí Lustig sí acepta que $\ddot{\mathrm{k}}>0$ por la cual $\ddot{\mathrm{k}}>\ddot{\mathrm{I}}$.

c) Cuando $\ddot{I}>0$, es cierto que " $\mathrm{d}^{2} \mathrm{k} / \mathrm{dt}^{2}$ puede ser mayor, menor o igual que $\mathrm{d}^{2} \mathrm{I} / \mathrm{dt}^{2} " .{ }^{23}$ Sin embargo, el asunto no tiene la indeterminación que alega Lustig porque cuando $\mathrm{I} \longrightarrow 0+$ $\dddot{\mathrm{k}}>$ İ y sigue siéndolo hasta un punto determinado que se calcula en la siguiente sección.

Antes de dejar el trabajo de Lustig, quisiera señalar otras confusiones en su manejo del cálculo. En la nota de pie de página No. 13. Lustig presenta un ejemplo artimético para aclarar la identificación de la desigualdad $\ddot{\mathrm{k}} \gtrless \ddot{\mathrm{I}}$ y por ello escribe cuatro supuestos:

1) $\mathrm{f}^{\prime}(\mathrm{k})+\mathrm{g}^{\prime}(\mathrm{k})+1=2$

2) $\mathrm{d}^{2} \mathrm{k} / \mathrm{dt}^{2}=5$

3) $f^{\prime \prime}(k)+g '(k)=-2$

4) $\mathrm{dk} / \mathrm{dt}=3$.

20 No encuentro esta homilía en el libro de Sweezy.

21 Lustig, p. 157.

22 ibid., p. 162.

23 ibid., p. 169. 
Luego, Lustig sustituye estos valores en su ecuación (8) para ilustrar que aunque $|\ddot{\mathrm{k}}|>|\ddot{\mathrm{I}}|, \dot{\mathrm{c}}$ " tiene signo negativo a pesar de que el ingreso aumente a una tasa creciente $\left(\mathrm{d}^{2} \mathrm{I} / \mathrm{dt}^{2}>0\right) " .{ }^{24}$ Así, Lustig toma como su ejemplo precisamente el caso excluido por Sweezy (I $>0$ ). A pesar de eso, el sistema de Lustig no resiste un examen crítico.

Existen tres maneras de interpretar las cuatro ecuaciones: Primero, puede dárseles la interpretación de funciones $f(k), g(k)$ y $k(t)$ que están evaluadas para alguna $k$, tal que en ese punto de tiempo exite una ko que nos da las cuatro ecuaciones. En este caso, Lustig necesita proveernos con las funciones explícitas $f(k), g(k)$ y $k(t)$ compatibles con las hipótesis de Sweezy y a la vez con sus cuatro ecuaciones. Es fácil mostrar que existen funciones explícitas que refuten las matemáticas de Lustig. Por ejemplo, considera las funciones,

$$
\begin{aligned}
& f(k)=\{1-\exp (-2 k)\} / 2 \\
& g(k)=\{1-\exp (-4 k)\} / 4 \\
& k(t)=\exp (\cdot 1 t) .
\end{aligned}
$$

Entonces, se puede calcular el valor de cada una de las cuatro ecuaciones para cualquier $t$. En particular, podemos tomar $t=0$, por lo cual

$$
\begin{aligned}
& \mathrm{f}^{\prime}(\mathrm{k})+\mathrm{g}^{\prime}(\mathrm{k})+1=1.15366 \\
& \mathrm{~d}^{2} \mathrm{k} / \mathrm{dt}^{2}=0.01 \\
& \mathrm{f}^{\prime \prime}(\mathrm{k})+\mathrm{g} \prime(\mathrm{k})=-.3439324 \\
& \mathrm{dk} / \mathrm{dt}=0.1 \quad \text { y por lo tanto, } \\
& \ddot{\mathrm{I}}=-0.022856<\ddot{\mathrm{k}} \text { y } \dot{\mathrm{c}}<0 \text { mientras que } \\
& \ddot{\mathrm{k}}>0 \text { dentro del rango que trata Sweezy }(\ddot{\mathrm{I}} \leq 0) .
\end{aligned}
$$

La segunda manera de interpretar las cuatro ecuaciones de Lustig es suponer que son relaciones funcionales y no funciones evaluadas en un punto dado. En este caso, el lector puede verificar que dada la

24 ibid., p. 163. 
ecuación 1), no queda Lustig en libertad a escoger según su gusto la ecuación 3 ), porque si

$$
\begin{aligned}
& f^{\prime}(k)+g^{\prime}(k)+1=2, \text { entonces } \\
& f^{\prime \prime}(k)+g^{\prime \prime}(k)=0
\end{aligned}
$$

Asimismo, si $\mathrm{d}^{2} \cdot \mathrm{k} / \mathrm{dt}^{2}=5, \mathrm{dk} / \mathrm{dt}$ no puede ser 3 sino tiene que ser $\left(5 t+c_{1}\right)$, donde $c_{1}$ es una constante arbitraria. Lustig calcula $\ddot{I}=4$, cuando debe ser $10>5=\ddot{\mathrm{k}}$, por lo cual c tiene signo positivo que en mi estimación es perfectamente compatible con una aceleración del ingreso nacional. Aparte de equivocarse en el cálculo, como ya hemos mencionado, Lustig olvida que $\ddot{\mathrm{I}}>0$ es precisamente el caso excluido por Sweezy por lo cual ella no puede usarlo y a la vez calificar como "internamente inconsistente" al modelo de Sweezy.

La tercera manera de interpretar las cuatro ecuaciones de Lustig, es que el lado derecho consiste de números cualesquiera no relacionados los unos con los otros. Esto presupone una desvinculación de las ecuaciones unas de otras por parte de Lustig y una respuesta por su parte podía esclarecer sus intenciones.

Después de sus nuevas matemáticas, Lustig concluye correctamente que: "Esta revisión de la lógica del desarrollo matemático del modelo pudo resultar tediosa" e incorrectamente que su revisión "ha permitido poner de manifiesto la inutilidad de este modelo como formulación rigurosa de la teoría de subconsumo de Sweezy". ${ }^{24}$ Lustig corona sus esfuerzos algebraicos con más comentarios que creemos irrelevantes para recomponer el modelo de Sweezy.

Nos queda la obligación de mostrar la proposición de Sweezy del estancamiento de la economía capitalista madura $(\mathrm{I} \leq 0) .{ }^{25}$ De hecho, mostraremos que aunque $\ddot{\mathrm{D}}>0$ en algún rango cerca de cero, las observaciones por parte de Sweezy son atinadas.

\section{Las condiciones para que $\ddot{k}>\ddot{I}$}

Existen varias maneras de establecer cuando $\ddot{k}>\ddot{I}$ y todas ellas dependen de la concavidad de $\mathrm{I}(\mathrm{k})$. En la primera parte de esta sección, comprobamos que cuando los cambios son discretos, la aceleración de $I$ es menor que la $k$ cuando la primera no es positiva. En la segunda parte de esta sección, verificamos que la tasa de crecimiento

\footnotetext{
25 Aunque es necesaria, la condición $\ddot{I}>0$ no garantiza una tasa creciente del ingreso nacional para la cual se requiere que $\ddot{\mathrm{I}} / \mathrm{I}>\mathrm{I} / \mathrm{I}>0$ cuando $\mathrm{i}>0$.
} 
de k supera a la de I con el crecimiento de la economía, es decir, para mayor k. Luego, en la tercera parte se establecen los requerimientos de tiempo y de consumo para que la aceleración de $\mathrm{k}$ sea igual a la de I como una línea que divide los casos de Sweezy de los excluidos por él. Finalmente en la cuarta parte, presentamos la comprobación matemática de la conclusión de Sweezy: $\ddot{\mathrm{k}}>\mathrm{I}$ cuando $\ddot{\mathrm{I}} \leqslant 0$.

\section{1) La relación entre la aceleración de $\mathrm{k}$ y la de $\mathrm{I}$}

En el Diagrama 1 se presentan las posibles gráficas de $f(k)$ y $g(k)$. Es posible que $f(0), g(0)>0$ por existir un límite inferior al consumo de los trabajadores y de los capitalistas respectivamente. En tal caso, $I(0)>0$ pero $I(k)$ sigue siendo cóncava y en base a esta propiedad de

\section{Diagrama 1}

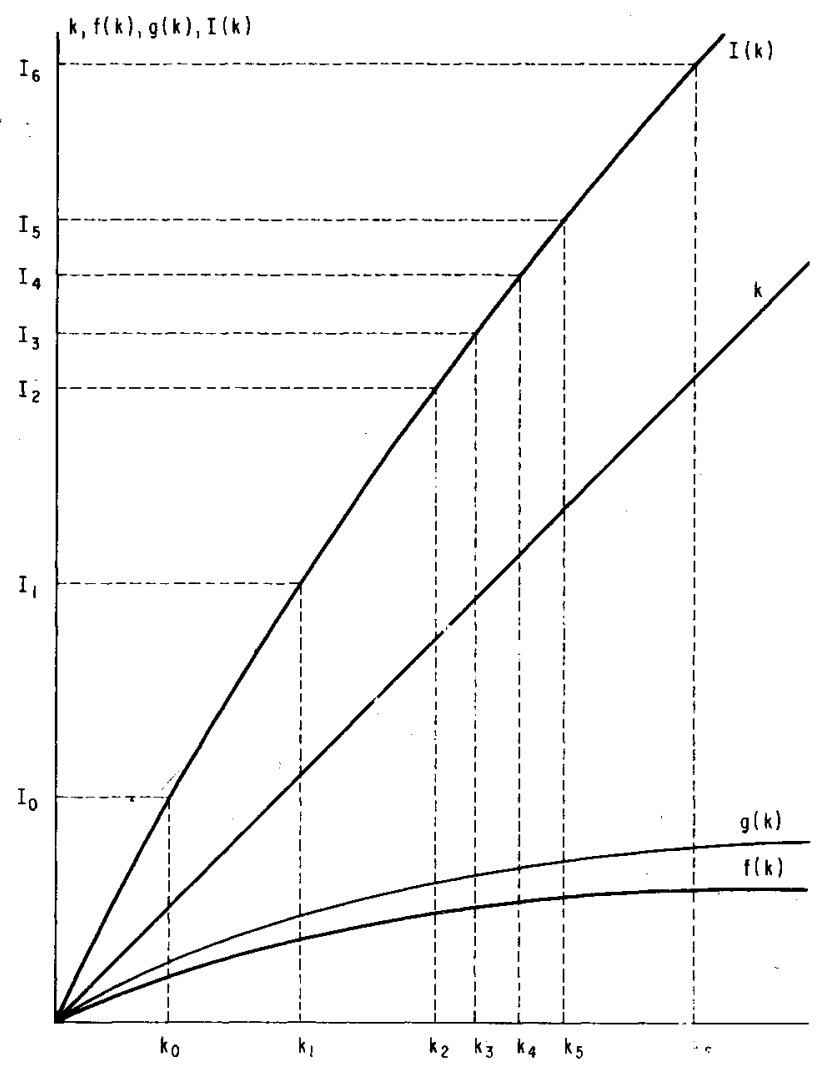


I(k) se establece la relación entre $\ddot{\mathrm{I}}$ y $\ddot{\mathrm{k}}$ cuando los cambios son discretos. Para simplificar, escogemos los cambios de tiempo $\left(\Delta t_{i}\right)$ igual a la unidad y a la vez hacemos iguales los cambios de $\mathbf{k}\left(\Delta \mathrm{k}_{\mathbf{i}}=\Delta \mathrm{k}_{\mathbf{j}}\right)$ para todos los números enteros, i, j. Así,

$$
\begin{aligned}
& \frac{\Delta \mathrm{k}_{0}}{\Delta \mathrm{t}_{0}}=\mathrm{k}_{1}-\mathrm{k}_{0}=\Delta \mathrm{k} ; \mathrm{y} \text { también } \\
& \frac{\Delta \mathrm{k}_{1}}{\Delta \mathrm{t}_{1}}=\mathrm{k}_{2}-\mathrm{k}_{1}=\Delta \mathrm{k} .
\end{aligned}
$$

Ahora, la aceleración de $\mathrm{k}$ con respecto al tiempo,

$$
\frac{\Delta^{2} \mathrm{k}_{0}}{\Delta \mathrm{t}_{0}^{2}}=\frac{\Delta \mathrm{k}_{1}}{\Delta \mathrm{t}_{1}}-\frac{\Delta \mathrm{k}_{0}}{\Delta \mathrm{t}_{0}}=0 .
$$

¿Qué pasa con II mientras que $\ddot{\mathrm{k}}=0$ ?

$$
\frac{\Delta^{2} I_{0}}{\Delta t_{0}^{2}}=\left(I_{2}-I_{1}\right)-\left(I_{1}-I_{0}\right)<0
$$

por la concavidad de I.

$$
\text { Es decir, cuando } \ddot{\mathrm{k}}=0, \ddot{\mathrm{k}}>\ddot{\mathrm{I}} \text {. }
$$

Ahora, consideremos la situación cuando $\ddot{\mathrm{k}}<0$, como pasa entre los tiempos 2 y 3 , suponiendo que el avance de $k$ es igual entre $t=2$ y $\mathrm{t}=5$.

$$
\begin{aligned}
& \frac{\Delta \mathrm{k}_{1}}{\Delta \mathrm{t}_{1}}=\mathrm{k}_{2}-\mathrm{k}_{1}=\Delta \mathrm{k}, \text { mientras que } \\
& \frac{\Delta \mathrm{k}_{2}}{\Delta \mathrm{t}_{2}}=\frac{\mathrm{k}_{5}-\mathrm{k}_{2}}{3}=\frac{\Delta \mathrm{k}}{3} \\
& \therefore \frac{\Delta^{2} \mathrm{k}_{1}}{\Delta \mathrm{t}_{1}^{2}}=\frac{\frac{\Delta \mathrm{k}}{3}-\Delta \mathrm{k}}{\Delta \mathrm{t}_{1}}=-\frac{2}{3} \Delta \mathrm{k}<0 .
\end{aligned}
$$


Mientras, ¿cómo responde Ï?

$$
\frac{\Delta^{2} \mathrm{I}_{1}}{\Delta \mathrm{t}_{1}^{2}}=\Delta \mathrm{I}_{2}-\Delta \mathrm{I}_{1}
$$

Por la concavidad de I, $3 \Delta \mathrm{I}_{2}+\epsilon=\Delta \mathrm{I}_{1}, \epsilon>0$

$$
\therefore \frac{\Delta^{2} I_{1}}{\Delta t_{1}^{2}}=-\frac{\left(2 \Delta I_{1}+\epsilon\right)}{3}
$$

lo cual es más negativo que

$$
\frac{\Delta^{2} k_{1}}{\Delta t_{1}^{2}}
$$

de nuevo, cuando $\ddot{\mathrm{k}}<\mathrm{o}, \ddot{\mathrm{I}},<\ddot{\mathrm{k}}$.

Ahora consideramos que $k$ avanza de $k_{4}$ a $k_{6}$ en tiempo 4 a 6 .

$$
\begin{aligned}
\therefore \quad \frac{\Delta k_{4}}{\Delta t_{4}} & =k_{5}-k_{4}=\frac{\Delta k}{3} ; \\
& \frac{\Delta k_{5}}{\Delta t_{5}}=k_{6}-k_{5}=\Delta k \\
\therefore \quad \frac{\Delta^{2} k_{4}}{\Delta t_{4}{ }^{2}} & =\Delta k-\frac{\Delta k}{3}=\frac{2}{3} \Delta k .
\end{aligned}
$$

La aceleración de I del tiempo 4 a 6 nos da,

$$
\frac{\Delta^{2} I_{4}}{\Delta t_{4}{ }^{2}}=\Delta I_{5}-\Delta I_{4}=I_{6}-2 I_{5}+I_{4}
$$

Pero mientras que $\left(I_{6}-I_{5}\right)>\left(I_{5}-I_{4}\right)$, también es cierto que 3 $\left(\mathrm{I}_{5}-\mathrm{I}_{4}\right)=\left(\mathrm{I}_{6}-\mathrm{I}_{5}\right)+\delta, \delta>0$.

$$
\therefore \quad \frac{\Delta^{2} \mathrm{I}_{4}}{\Delta \mathrm{t}_{4}{ }^{2}}=\frac{2}{3} \Delta \mathrm{I}_{5}-\frac{\delta}{3} \cdot
$$

Ahora bien, mientras que $\Delta \mathrm{I}_{5}>\Delta \mathrm{k}$, a la vista no existe ninguna seguridad acerca del tamaño de $\delta$.

$$
\therefore \quad \frac{\Delta^{2} k_{4}}{\Delta t_{4}{ }^{2}} \quad \geqslant \quad \frac{\Delta^{2} I_{4}}{\Delta t_{4}{ }^{2}} \cdot
$$


Es decir, cuando hacemos el cambio de tiempo muy pequeño y lle- . gamos a la continuidad de las variables,

$$
\ddot{\mathbf{k}} \geqslant \ddot{\mathrm{I}} \text { cuando } \ddot{\mathbf{k}}>\mathbf{0} \text {. }
$$

La conclusión de un examen con cambios discretos de una función cóncava como la planteada por Sweezy, es que si $\ddot{I} \leqslant 0, \ddot{\mathbf{k}}>\ddot{\mathrm{I}}$. Esto coincide perfectamente con la conclusión de Sweezy.

2) La relación entre las tasas de crecimiento de $\mathrm{k}$ y de I para una economía en crecimiento

En esta parte damos un ejemplo gráfico con funciones explícitas de $f(k)$ y $g(k)$, con el objeto de establecer que la tasa de crecimiento de $k$ supera a la de I para una economía que crece $(k>0)$.

Para mostrar esta proposición tenemos 3 gráficas en el Diagrama 2, dibujados respetando las funciones explícitas.

$$
\begin{aligned}
& f(k)=2\{1-\exp (-k / 2)\} ; y \\
& g(k)=4\{1-\exp (-k / 4)\}
\end{aligned}
$$

En la primera parte del Diagrama 2, se ve que los valores de las funciones en orden descendente corresponden a $I, k, g$ y finalmente $f$ que también es el orden que siguen los aumentos absolutos de esas funciones causadas por un aumento de $k$, como se observa en la segunda parte del Diagrama. En la tercera parte del Diagrama, se presentan los aumentos en cada variable por cada unidad extra de $\mathrm{k}$ relativamente a la variable misma y se ve que $k^{\prime} / k=1 / k>I^{\prime} / I$ e incluso $f^{\prime} / f \circ$ g'/g (pero no los dos) pueden exceder a I'/I.

Ahora, consideremos un sistema que crece en el tiempo que en nuestro caso implica que $\dot{k}>0$. Multiplicando los valores de la tercera parte del Diagrama 2 por $k$ nos dará $\mathrm{k} / \mathrm{k}, \mathrm{I} / \mathrm{I}, \mathrm{l} / \mathrm{f}$ y $\mathrm{g} / \mathrm{g}$ que son tasas de crecimiento de las cuatro variables. Es claro que el orden se mantiene dependiente del signo de $k$ y si ésta es negativa, la tasa de crecimiento (negativo) de $\mathrm{k}$ será menor que las de $\mathrm{I}, \mathrm{f}$ y g.

Además, si $\mathrm{k}$ es mayor, la diferencia relativa entre las diferentes tasas de crecimiento es mayor, como se aprecia en la tercera parte del Diagrama 2, en la cual, 


\section{Diagrama 2}
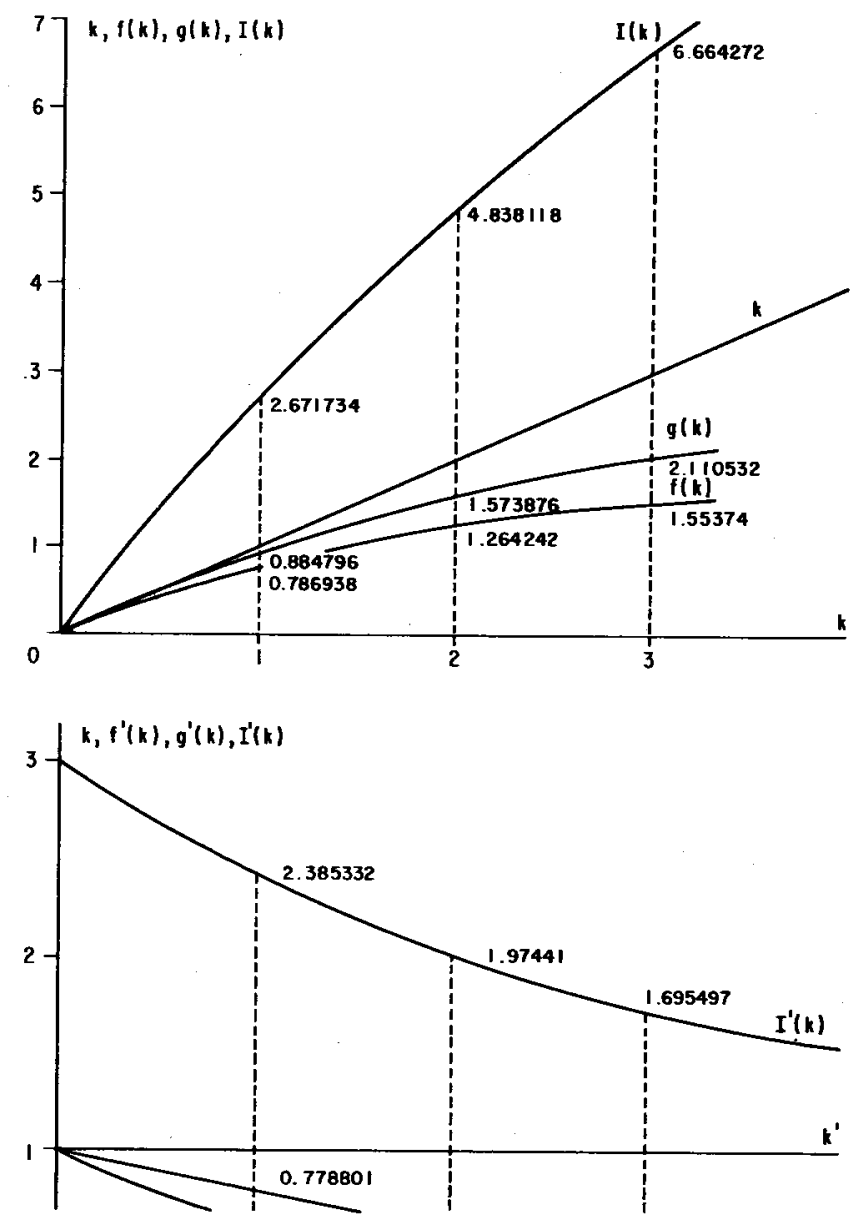

$$
\left.\frac{k^{\prime} / \mathbf{k}}{I^{\prime} / I}\right|_{k=3}>\left.\quad \frac{k^{\prime} / k}{I^{\prime} / I}\right|_{k=2}>\left.\quad \frac{k^{\prime} / k}{I^{\prime} / I}\right|_{k=1,} \text {, etc. }
$$

podemos asegurar lo mismo con referencia a los cambios respecto al tiempo, por lo cual 


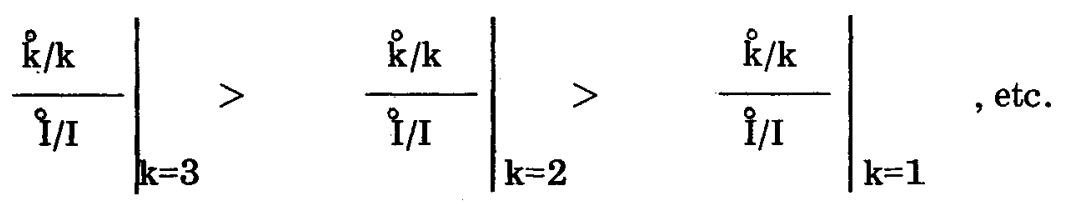

sin considerar el signo de $\mathbf{k}$.

\section{3) El impacto del tiempo sobre el consumo}

Establecemos ahora una relación más formal entre $\ddot{\mathrm{k}}$ e $\ddot{\mathrm{I}}$ que incluye explícitamente el factor tiempo en el movimiento del sistema.

Empezamos con la identidad,

$$
I \equiv x+k, \quad x \equiv f(k),+g(k)
$$

que derivamos dos veces con respecto a tiempo para obtener

$$
\ddot{\mathrm{I}}=\left(\mathrm{x}^{\prime}+1\right) \ddot{\mathrm{k}}+\mathrm{x}^{\prime \prime}(\mathrm{k})^{2} \text {. }
$$

¿Cuáles son los valores de $\mathrm{k}$ y $\mathrm{t}$ por los cuales $\ddot{\mathrm{I}}=\ddot{\mathrm{k}}$ ?

Si $\ddot{\mathrm{I}}=\ddot{\mathrm{k}}$, la ecuación diferencial anotada arriba se convierte en solamente una variable, $k$. Tenemos pues,

$$
x^{\prime} \ddot{k}+x^{\prime \prime}(\mathfrak{k})_{2}=0 .
$$

El lector puede verificar que,

$$
x(k)=c_{1} t+c_{2}
$$

es la solución general de la ecuación diferencial, ${ }^{26}$, donde $c_{1}, c_{2}$, son constantes que se determinan solamente cuando tenemos más información acerca de la ubicación de $\mathrm{k}$ en dos puntos de tiempo.

El significado de la solución a la ecuación diferencial es lo siguiente: Con el pasaje del tiempo,

$$
x(k)<c_{1} t+c_{2}
$$

26 Dividiendo la ecuación diferencial entre $\mathrm{kx}$ ' e integrando dos veces con respecto a tiempo. 
por la concavidad de $f(k)$ y $g(k)$ y por lo tanto $x(k)$ y el avance del tiempo.

En esta situación, se puede comprobar que la ecuación diferencial entre $\ddot{\mathrm{k}}$ e $\ddot{\mathrm{I}}$ se mantiene si y solamente si $\ddot{\mathrm{k}}>\ddot{\mathrm{I}}$.

Damos un ejemplo con condiciones iniciales $k(0)=0 ; k(1)=1 \mathrm{y}$ aprovechando las funciones explícitas usadas anteriormente en el Diagrama 2, tenemos

$$
c_{1}=1.6717 ; c_{2}=0 \text {. }
$$

Realmente, si $f(0)+g(0)=k(0)=0$, entonces $c_{2}=0$ como en nuestro ejemplo.

Es decir, para una economía que empieza sin plusvalía, que tiene una unidad de plusvalía en el primer periodo de tiempo y con relaciones de consumo como las dadas, $\ddot{\mathrm{k}}=\ddot{\mathrm{I}}$ cuando se mantiene la ecuación.

$$
6-2 \exp (-\mathrm{k} / 2)-4 \exp (-\mathrm{k} / 4)=1.6717 \mathrm{t} .
$$

Es obvio que para que esta igualdad pueda mantenerse es necesario que $t \leqslant 3.59$. Aún en este tiempo, es posible que la ecuación no se mantenga si $\mathbf{k}$ no aumenta rápidamente. Por ejemplo, se ve que la igualdad falla si $k=2$ cuando $t=2$. Luego $\ddot{k}>I$ como en el ejercicio anterior y se ve de nuevo que la conclusión de Sweezy está apoyada por las matemáticas.

\section{4) $\underline{\ddot{\mathrm{k}}}>\ddot{\mathrm{I}}$ cuando $\ddot{\mathrm{I}} \leqslant 0$.}

Empezamos con la ecuación (8) de Sweezy:

$$
\ddot{I}=\left(f^{\prime}+g^{\prime}+1\right) \ddot{k}+\left(f^{\prime \prime}+g^{\prime \prime}\right) \cdot\left(k^{2}\right)^{2} \text {. }
$$

Además, en cualquier momento de tiempo $t$, existe una $k$. $k$, funciones de $t$, tal que existe la línea recta.

$$
\ddot{\mathbf{I}}=\mathbf{a}+\mathbf{b} \ddot{\mathbf{k}}
$$

en las variables $(\ddot{k}, \ddot{I})$ en donde

$$
\begin{aligned}
& \mathrm{a} \equiv\left\{\mathrm{f}^{\prime \prime}(k)+\mathrm{g}{ }^{\prime \prime}(k)\right\}(\mathrm{k})^{2} ; \mathrm{y} \\
& \mathrm{b} \equiv \mathrm{f}^{\prime}(k)+\mathrm{g}^{\prime}(k)+1
\end{aligned}
$$


son los parámetros de una línea recta que representa la relación entre $\ddot{\mathrm{k}}$ e $\ddot{\mathrm{I}}$ en cualquier momento dado. Tenemos $3>\mathrm{b}>1$ tal que la línea tiene pendiente mayor que 1 , además a es negativa para dar la línea dibujada en el Diagrama 3.

\section{Diagrama 3}

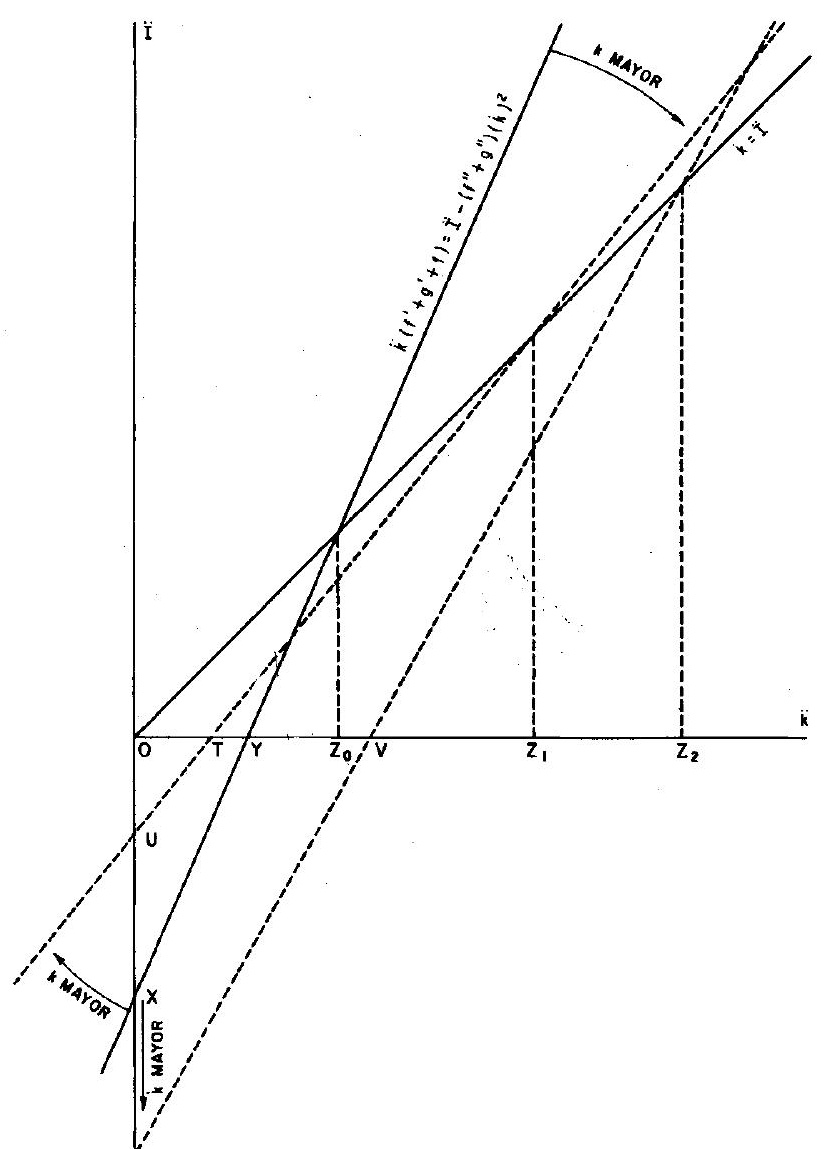


En adición $\partial \mathrm{b} / \partial \mathrm{k}<0$, por lo cual para mayor $\mathrm{k}^{27}$ tenemos menor b y menor pendiente de la línea $\overrightarrow{\mathrm{I}}=\mathrm{a}+\mathrm{b} \ddot{\mathrm{k}}$. $\mathrm{El}$ mismo resultado se obtiene considerando que $\partial \mathrm{b} / \partial \mathrm{t}<0 \mathrm{y}$ por lo tanto la pendiente de la línea se reduce con el pasaje del tiempo. Los dos efectos aumentan el rango del estancamiento de la economía capitalista, incluyendo así una parte creciente del área $(\ddot{\mathrm{k}}, \ddot{\mathrm{I}})>0$ dentro del área de estancamiento.

Para medir el efecto de $\mathrm{k}$ sobre a dentro del rango que nos interesa $(\ddot{\mathrm{k}}<0)$, obsérvese que $\stackrel{\mathrm{k}}{\mathrm{a}} 0$ y creciendo, además, $\partial \mathrm{a} / \partial \mathrm{k}<0$, por lo tanto en el rango $\mathrm{k}>0_{2 .}$ a será cada vez más negativa y en estos casos la línea que relaciona $\mathrm{k}$ a $\ddot{\mathrm{I}}$ mueve hacia los puntos $\mathrm{V}, \mathrm{W}$ y aumenta el rango de posibilidades para el estancamiento de la economía capitalista. Es decir, un aumento de la plusvalía invertida, Ceteris Paribus, implica una mayor área de estancamiento de la economía capitalista exactamente como la describió Sweezy.

Existe la posibilidad de que $\mathrm{k}$ aumente y $\mathrm{k}$ disminuya. Es decir, que $\ddot{\mathbf{k}}<0$. En este caso es posible que se reduzca el área de estancamiento (como se muestra en el Diagrama 3), con la línea que pasa por los puntos U, T. Sin embargo, aunque se reduce el área de estancamiento, $\ddot{k}$ es negativo y mayor que $\ddot{I}$ en todo este rango. No se puede evitar la conclusión de que cuando la economía capitalista experimenta un crecimiento acelerado, la tendencia hacia el estancamiento es tanto mayor y cuando esa economía experimenta un crecimiento no acelerado, las fuerzas que lo estancan son más visibles, es decir, la tendencia al estancamiento está encarnada en el cuerpo de la economía capitalista.

\section{Observaciones sobre el apéndice}

Hemos mostrado que Sweezy tiene la razón y Lustig no la tiene en las primeras tres secciones de este artículo. En esta sección agregaremos unas observaciones al Apéndice del libro de Sweezy.

\section{1) La composición orgánica del capital}

¿Qué implica el modelo para la composición orgánica del capital, o en términos neoclásicos, de la profundidad del capital?

Derivamos la relación $(\mathrm{k} / \mathrm{I})$ una vez con respecto al tiempo, sustituyendo antes $(x+k)$ por $I$. Ahora $f, g$ y por lo tanto $x$, son funciones

27 Que es diferente de cuando $f$ crece. Este último implica que $t$ cambia, mientras que la suposición de mayor $k$, requiere que $t$ sea constante. 
cóncavas de tal suerte que para $\mathrm{k}$,

$$
\mathrm{f}>\mathrm{kf} \mathbf{f}^{\prime} ; \mathrm{g}>\mathrm{kg} ; \quad \mathrm{x}>\mathrm{kx}{ }^{\prime}{ }^{28}
$$

Así que $\dot{k} / \mathrm{I})>0$ cuando $\stackrel{\mathrm{k}}{>}>0$ e $\mathrm{I}$ crece menos en relación a $\mathrm{k}$. Este es el sistema que aumenta constantemente la composición orgánica del capital y produce la tendencia descendente de la tasa de ganancia con el paso del tiempo.

La misma demostración de cada vez mayor acumulación de plusvalía es posible, considerando una manipulación de la derivación de la identidad fundamental para dar:

$$
\frac{\mathrm{I}}{\mathrm{I}}=\left(\mathrm{x}^{\prime}+1\right) \frac{\mathrm{k}}{\mathrm{k}} \cdot \frac{\mathrm{k}}{\mathrm{k}}
$$

Ahora, la tasa de crecimiento de I es $\frac{>}{<}$ que la de k según como

$$
\left(x^{\prime}+1\right) k \stackrel{>}{\gtrless}(x+k) \text {. }
$$

Apelando a la concavidad de $\mathrm{x}$, tenemos que

$$
\frac{\stackrel{\circ}{\mathrm{I}}}{\mathrm{I}}<\frac{\stackrel{\circ}{\mathrm{k}}}{\mathrm{k}}
$$

como antes y esto asegura que $(\mathrm{k} / \mathrm{I})$ aumente sobre tiempo.

\section{2) El acelerador}

Sweezy utiliza el acelerador para dar una relación fina entre el consumo adicional $(\mathrm{x})$ y la inversión necesaria (c) para producir esos bienes en cada tiempo adicional (dt). Tal vez, la relación fija no es la más idónea a la luz del trabajo econométrico. Por las economías de escala y los cambios tecnológicos "embodied" y "disembodied", es más factible que $\lambda$ disminuya a través del tiempo.

28 Considérese una función cóncava como $\mathrm{f}(\mathrm{k})$ presentada en el diagrama 4.

$f^{\prime}(k)=C A / B C$ al punto $D$ y $k$ vale $B C$ por lo cual $k f^{\prime}(k)=C A<D A=f(k)$. Esta relación se mantiene para todas las funciones cóncavas, aun aquéllas con $f^{\prime}(k)>1$, mientras que $f(0) \geqslant 0$, y no creo que Sweezy argumentara que $f(0)<$ 0 . 


\section{Diagrama 4}

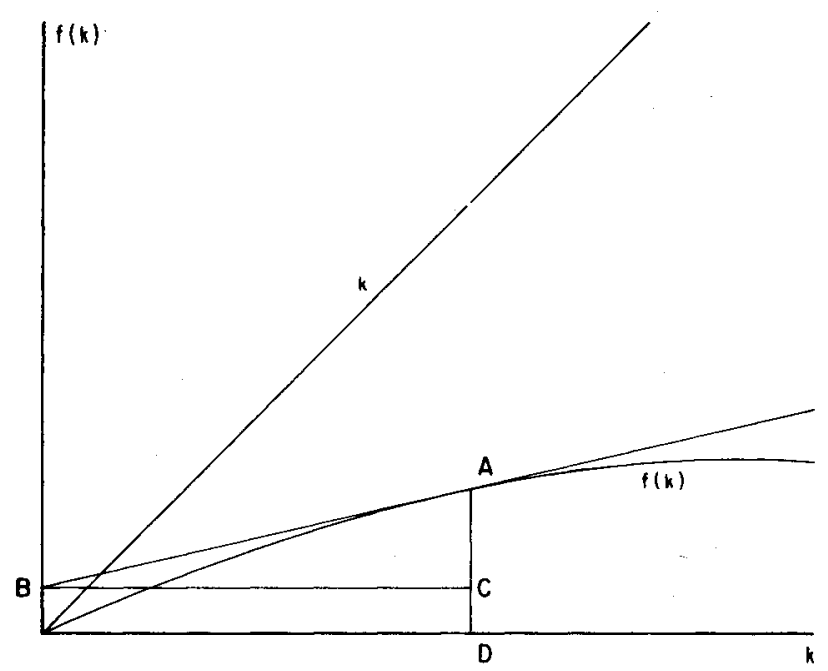

Así tenemos de Sweezy,

$c=\lambda \stackrel{\circ}{\mathbf{x}}=\lambda \mathbf{x}, \stackrel{\circ}{\mathbf{k}}$,

pero si la plusvalía nuevamente invertida $(\stackrel{\circ}{k})$ iguala a las nuevas necesidades de inversión, según el aumento en la demanda de consumo, debemos tener

$$
\stackrel{\circ}{\mathbf{c}}=\stackrel{\circ}{\mathbf{k}} \text {. }
$$

Es decir, otra manera para establecer un crecimiento de la plusvalía acumulada y (productivamente) invertida es exigir que

$$
\frac{\stackrel{\circ}{\mathrm{k}}}{\mathrm{k}}=\frac{\stackrel{\circ}{\mathrm{c}}}{\mathrm{c}}=\frac{1}{\lambda \mathrm{x}^{\prime}}
$$

que dice que la tasa de crecimiento de bienes de capital debería aumentar cada vez que aumente la de la plusvalía y como ésta sigue creciendo $^{29}$ también debería aumentar la tasa de crecimiento de bienes

$29 \underset{k \rightarrow \infty}{\operatorname{Lim} .} f^{\prime}(k), g^{\prime}(k) \rightarrow 0$. 
de consumo. Creo que en general la economía capitalista no exhibe esta característica.

El resultado es una crisis, que es la medicina para componer al sistema capitalista, bajando $\mathrm{k}$ hasta que las condiciones de recuperación prevalecen de nuevo, o en su ausencia, sigue el estancamiento.

$\mathrm{Si}$ adicionalmente, por los cambios tecnológicos o por la creciente escala, o por "Learning by Doing" 30 o cualquier otra razón, $\lambda$ empieza a disminuir, el punto de crisis (sobreproducción, subconsumo, disminución de la tasa de ganancia, aumento de la tasa de desempleo) llega más pronto y a un nivel de $\mathrm{k}$ menor que el caso en el cual $\lambda$ es fija.

En conclusión, la hipótesis del "coeficiente fijo" entre el consumo y la maquinaria necesaria para producirlo permite que la economia capitalista llegue más lentamente a la contradicción entre las dos tasas de crecimiento (de $\mathrm{k}$ por las necesidades de acumulación y de $\stackrel{c}{\mathrm{c}}$ por las necesidades de consumo). Como hemos observado antes, el área de estancamiento es mayor que lo que Sweezy estableció y partiendo de sus supuestos podría haber argumentado con más fuerza.

\section{3) El filo de navaja de Harrod}

La formulación de Sweezy podría parecer, si se tomara superficialmente, semejante al filo de navaja de Harrod.

La tasa de crecimiento de la plusvalía y de los bienes de capital debe de ser,

$$
\frac{1}{\lambda x^{\prime}} \text {. }
$$

Como $\mathrm{k}$ crece, estas tasas deben crecer y al mismo ritmo. Esta segunda condición puede ser satisfecha solamente por coincidencia accidental de las dos tasas. En este sentido, la tasa de crecimiento senalada es una línea divisoria entre la desproporcionalidad de la plusvalía y de los bienes de capital; sin embargo, el problema de la desproporcionalidad entre $\mathrm{k}$ y $\mathrm{c}$ está completamente superado por la tendencia de la primera a exceder a la segunda.

30 Véase Kenneth J. Arrow, "The Economic Implications of Learning by Doing", en Review of Economic Studies, Vol. XXIX, junio de 1962, pp. 155183 y la discusión subsiguiente en la literatura convencional. 


\section{4) El crecimiento del área de estancamiento}

En el Diagrama 3, la relación

$$
\frac{\mathrm{OZ}_{0}}{\mathrm{OY}}=1+\frac{1}{\mathrm{f}^{\prime}+\mathrm{g}^{\prime}}
$$

tal que cuando $\mathrm{k}$ es mayor, el área de estancamiento es mayor, que es precisamente la situación de la economía capitalista madura. ${ }^{31}$

Ahora bien, si para empezar, esta economía se encuentra momentáneamente a la derecha del punto $Z_{O}$, entonces $\stackrel{\circ}{c}>0$ y la. economía crece rápidamente. $\mathrm{El}$ aumento consecuente de $\mathrm{k}$ hace $\left(\mathrm{f}^{\prime}+\mathrm{g}^{\prime}\right)$ más pequeña y por lo tanto aumenta la diferencia entre los puntos $\mathrm{Z}_{\mathrm{O}}$ y $\mathrm{Y},{ }^{32}$ ampliando así el área de estancamiento. En el próximo período de tiempo, la línea que relaciona $\ddot{\mathrm{k}}$ a $\ddot{\mathrm{I}}$ se mueve hacia la derecha por lo cual $Z_{0}$ puede estar en $Z_{1}$ y por lo tanto tiende a empujar a la línea sólida tras el punto inicial de $\ddot{\mathrm{k}}$ que ahora podría quedar incluido dentro del área de estancamiento. Como mostraremos en las próximas páginas, no tiene sentido hablar del punto de equilibrio, de descanso, de la economía capitalista dentro del modelo de Sweezy, en el cual la única salida de la economía capitalista desarrollada para recuperar su camino normal hacia el estancamiento es la crisis.

\section{5) El filo de navaja de Sweezy}

Una manera de estudiar la posibilidad de un crecimiento ordenado de la economía capitalista, es examinar las condiciones que conducen a un crecimiento parejo de las principales variables de esa economia que son $\mathrm{I},(\mathrm{f}+\mathrm{g}), \mathrm{k}, \mathrm{y}$ c. Así, si r es la misma tasa de crecimiento de estas variables, tenemos:

$$
\mathbf{r}=\frac{\stackrel{\circ}{\mathrm{I}}}{\mathrm{I}}=\frac{\stackrel{\circ}{\mathrm{x}}}{\mathrm{x}}=\frac{\stackrel{\circ}{\mathrm{k}}}{\mathrm{k}}=\frac{\circ}{\mathrm{c}} ; \quad \mathrm{x} \equiv \mathrm{f}+\mathrm{g} .
$$

Es decir, las veredas que debe mantener la economía capitalista son:

$\mathrm{I}(\mathrm{t})=\mathrm{I}(0) \mathrm{e}^{\mathrm{rt}} ; \mathrm{x}(\mathrm{t})=. z(0) \mathrm{e}^{\mathrm{rt}}$, etc.

31 Roy F. Harrod, "An Essay in Dynamic Theory", The Economic Journal, Vol. XLIX, marzo de 1939, pp. 14-33.

32 El lector puede comprobar con las funciones $f$ y g especificadas antes, que cuando $\mathrm{k}=0$, la relación entre $\mathrm{Z}$ y $\mathrm{Y}$ es de 3 a 2 mientras que cuando $\mathrm{k} \rightarrow \infty \mathrm{la}$ relación hace la misma. 
Sustituyendo $\mathrm{e}^{\mathrm{rt}}$ en tres de estas unidades, tenemos:

$$
\begin{aligned}
& \mathrm{I}(\mathrm{t})=\mathrm{I}(0) \mathrm{k}(\mathrm{t}) / \mathrm{k}(0)=\alpha \mathrm{k}(\mathrm{t}), \alpha=\mathrm{I}(0) / \mathrm{k}(0) ; \\
& \mathrm{x}(\mathrm{t})=\mathrm{x}(0) \mathrm{k}(\mathrm{t}) / \mathrm{k}(0)=\beta \mathrm{k}(\mathrm{t}), \beta=\mathrm{x}(0) / \mathrm{k}(0) ; \mathrm{y} \\
& \mathrm{c}(\mathrm{t})=\mathrm{c}(0) \mathrm{k}(\mathrm{t}) / \mathrm{k}(0)=\gamma \mathrm{k}(\mathrm{t}), \gamma=\mathrm{c}(0) / \mathrm{k}(0)
\end{aligned}
$$

Es decir, el sistema se mueve al mismo ritmo que $\mathbf{k}$ y de acuerdo a su punto inicial $[\mathrm{I}(0), \mathrm{x}(0), \mathrm{k}(0), \mathrm{c}(0)]$.

Ahora bien, en el modelo de Sweezy,

$$
c=\lambda \stackrel{\circ}{\mathrm{x}}=\lambda \mathrm{rx}=\lambda \mathrm{r} \beta \mathrm{k} .
$$

Pero, para obtener un crecimiento ordenado, c debe ser siempre igual a $\mathrm{k}$ por lo cual,

$$
r=1 / \lambda \beta .
$$

Cualquier otra tasa de crecimiento será incompatible con un crecimiento ordenado de la economía capitalista y por lo tanto podemos considerar $r$ el filo de navaja de Sweezy, de tal suerte que cualquier desviación de una variable cualquiera de ese ritmo de crecimiento producirá una desviación cada vez mayor de las otras variables de la economía. La relación entre c y $\mathrm{x}$ es lineal, por lo cual otra condición para el crecimiento ordenado de esta economía capitalista, es que la función de consumo global x(I) sea lineal. De hecho, hay bastante evidencia econométrica a favor -como en contra- de esa suposición, según el plazo de tiempo considerado; sin embargo, me parece una relación demasiado estrecha para una economía en todo tiempo.

La conclusión de esta sección es, que solamente por accidente se logrará un crecimiento ordenado de la economía capitalista madura y que normalmente ésta reflejará una anarquía en cualquier momento de tiempo dado. Una condición necesaria para obtener una "edad de oro" exige linealidad entre $x$ y $k(x "=0)$, mientras que la hipótesis de Sweezy (también de Keynes) es que $x "<0$ y dentro de este modelo no existe ningún punto de equilibrio sino una anarquía perpetua estimulada por condiciones coyunturales y siempre tendiendo al estancamiento. 


\section{6) La síntesis neoclâsica}

El mundo neoclásico no acepta ni el "filo de navaja" de los postkeynesianos, ni las ideas del "estancamiento" de Sweezy, ni el de otros marxistas y no marxistas como Hansen. ${ }^{33}$ Que $\mathrm{k} / \mathrm{k}>\mathrm{I} / \mathrm{I}$ siempre, parece una conclusión herética para la sabiduría neoclásica establecida.

El modelo expuesto por Sweezy contrasta con la imaginación neoclásica de la realidad capitalista. La edad de oro de Phelps ${ }^{34}$ con el pleno empleo del capital y de la fuerza de trabajo y con un crecimiento relativo de las principales variables al mismo ritmo (el producto per-capita y la relación capital trabajo), no se produce sin las inconsistencias anotadas por la escuela de Cambridge de Joan Robinson, ${ }^{35}$ el regreso de Ricardismo y la teoría del valor-trabajo en Piero Sraffa, ${ }^{36}$ el esfuerzo posterior por construir alguna línea de defensa seguido por las nuevas inconsistencias de los neoclásicos ${ }^{37}$ y los últimos golpes de una gran variedad de autores ${ }^{38}$ que han producido el derrumbe del neoclasicismo.

Más de un siglo después de la aparición de los primeros monopolios ferrocarrileros de los E.E.U.U, las hipótesis competitivas del neoclasicismo son difíciles de defender. Solamente los fieles siguen los rituales y me parece que actualmente hay más receptores de esas ideas conservadoras en la Periferia que en el Centro productor de las mismas, donde son lógicamente insostenibles. Lo mismo se puede decir del Keynesianismo, del monetarismo, del estructuralismo y de otras corrientes. En contraste, las ideas de Sweezy y el Apéndice son

33 Alvin H. Hansen, "Economic Progress and Declining, Population Growth", The Américan Economic Review, Vol. 29, marzo de 1939, pp. 1-15.

34 Phelps, E.S., “The Golden Rule of Acumulation: A Fable for Growthmen", The American Economic Review Vol. 51 No. 4, septiembre de 1961.

35 A fines de los años sesenta, hubo un intercambio transatlántico sostenido entre los dos Cambridge con la inglesa saliendo victoriosa. De los varios escritos de Joan Robinson, remito al lector a "La función de producción y la teoría del capital" en Teoría del Desarrollo, Aspectos Críticos, Ed. Martínez Roca, Barcelona, 1973.

36 Piero Sraffa, Production of Commodities by Means of Commodities, Cambridge University Press, London, 1960.

37 Para un resumen de estos esf́uerzos, véase Pierre Salama, Sobre el Valor, Ediciones Era, S. A., México, 1978, pp. 88-118.

38 Véase, por ejemplo, los varios trabajos de Edward Nell, Luigi Pasinetti, Pierangelo Garegnani, A. Bhaduri y A. Shaikh entre otros, sobre las múltiples deficiencias e inconsistencias de la escuela neoclásica. 
claros en sus conclusiones, corroboradas por una larga y creciente crisis actual cuya solución no se vislumbra ni en el plano teórico de los economistas burgueses y consecuentemente menos aún en el plano de las acciones políticas, reflejadas a través del malestar sentido por los principales hechiceros Keynesianos de nuestros días.

\section{7) Tres críticas y una adición}

El modelo de Sweezy ha sido criticado con mayor justicia desde el punto de vista de que las conclusiones están encarnadas en los supuestos del modelo. Mandel dice: "Son siempre peticiones de principio, que suponen ya demostrado en el enunciado del problema, la solución que quieren aportar". ${ }^{39}$

Por otra parte, Shaikh llega a la conclusión de que "El error fundamental en el análisis de Sweezy es el tradicional error subconsumista de reducir el Departamento I al papel de un 'insumo' en el Departamento II". ${ }^{40}$ Pero es posible que los bienes de capital produzcan otros bienes de capital. Es más que posible como explica Shaikh, es parte de la naturaleza de la reproducción ampliada. Es decir, el concepto de acelerador no es el indicado para captar la serie de bienes de producción en la cual cada generación depende de la generación anterior y solamente la última de éstas dependerá del consumo y posiblemente de sus aumentos y de los aumentos de estos, etc.

Cuando se considera el subconsumismo no es posible ignorar el trabajo de Michael Bleaney. ${ }^{41}$ Además, Lustig y Shaikh en sus artículos respectivos aprueban sus argumentos, categorías y opiniones y por lo tanto la crítica del modelo de Sweezy por parte de Bleaney merece nuestra atención. ${ }^{42}$ En su capítulo VI, dedicado a la posición de Carlos Marx respecto al subconsumo, Bleaney concluye lo siguiente: "Entonces la concepción de Marx de una economía capitalista es muy diferente de la de los subconsumistas. Además, sus esquemas de la reproducción, que son un avance teórico definitivo con respecto a

39 Ernest Mandel, Tratado de Economía Marxista, Ediciones Era, S. A., México, 1969, Tomo I, p. 341.

40 Anwar Shaikh, "Introducción a la Historia de las Teorías de la Crisis", Investigación Económica, 145, julio-septiembre 1978, p. 133.

41 Bleaney, Michael, Teorías de las Crisis, Editorial Nuestro Tiempo, México, 1977.

42 Ibid., pp. 142-153, con una repetición desafortunada de las últimas páginas. 
lo producido por sus predecesores, muestran claramente los errores de los argumentos de Sismondi y Malthus. Esto debió ser tenido en cuenta por Sweezy, cuando examinó párrafos aislados y los presentó como evidencia de que Marx tenía una teoría subconsusmista de la crisis". ${ }^{43}$ Bleaney dedica las páginas siguientes a subestimar las citas de Marx en el libro de Sweezy e intenta separarlos. Luego, critica el modelo de Sweezy que es lo que nos concierne aquí. Repetimos sus dos críticas.

Primero, "El error fundamental que Sweezy ha cometido, en realidad, consiste en suponer que debido a la lógica del bienestar humano la inversión no se justifica si no conduce al aumento del consumo; esto también debe ser cierto para la producción capitalista, la cual no puede persistir durante mucho tiempo con una tasa de inversión (fuera de línea) con su demanda de consumo. ${ }^{44}$

Es decir, que la función del capital no es solamente física (producir bienes de consumo o más bien de producción), sino también social (acumulación por parte de la clase capitalista).

"En segundo lugar, como todos los otros subconsumistas -excepto en cuanto a que ellos descuidan mencionar el problema en su conjunto-Sweezy tiene que explicar por qué la inversión no puede llenar el vacío en la demanda efectiva dejada por el consumo". ${ }^{45}$

Este es el argumento de Shaikh y claramente es válido. "Las máquinas pueden ser usadas para producir máquinas", ${ }^{45}$ aumentando así la productividad del trabajador y el ingreso nacional constantemente.

Básicamente, las críticas de Bleaney se reducen al establecimiento de una mayor demanda para los bienes de producción independientemente de la problemática de la subproducción/subconsumo que un "exceso" de bienes de producción podia crear.

Desde nuestro punto de vista, el modelo de Sweezy contiene dos de los tres elementos para explicar el proceso de crecimiento de la economía capitalista. Estos dos elementos son las relaciones de $x(k)$ $\mathrm{y}$ el aumento de los bienes de producción $\lambda \stackrel{\circ}{\mathrm{x}}$. Creemos que la tercera relación es entre c y la producción resultante, I. Igualmente sentimos la rigidez de las dos relaciones principales de Sweezy: por una parte el acelerador no es un concepto adecuado para examinar la demanda para los bienes de producción y por otra parte la concavidad de $\mathrm{x}$ con respecto a $\mathrm{k}$ es demasiado estrecha y de hecho, su división en dos ti- 
pos de consumo facilita que la relación de uno de estos con el ingreso total pueda subir con un aumento del ingreso. ${ }^{46}$ Creemos que es posible completar el modelo de Sweezy sin estos problemas que consideramos al margen del modelo. En este artículo no intentamos esta tarea por considerar que nos alejaría bastante del propósito inicial que îue mostrar las equivocaciones de Lustig; sin embargo, proponemos establecer en otro artículo las condiciones necesarias y suficientes para el estancamiento de la economía capitalista.

Sin tratar de minimizar las deficiencias ya señaladas, vale la pena recordar que, desde cierto punto de vista, Sweezy simplemente adoptó las dos ideas importantes de los años treinta: ${ }^{47}$ el multiplicador, basado en funciones de consumo, explícitamente cóncavas y operantes en el rango del ingreso correspondiente a menos de "pleno empleo" y el acelerador, una función lineal de inversión, operante en el rango de ingreso correspondiente al "pleno empleo". Lógicamente no podemos tener las dos situaciones a la vez, sin embargo, Samuelson, Phillips, Hicks y otros ${ }^{48}$ combinaron los dos conceptos para producir una serie de modelos de la "interacción" del multiplicador y del acelerador, variando los tiempos de respuesta del consumo y/o de la inversión a cambios del ingreso y llegando a ilustrar el efecto de los dos parámetros (el de consumo/ahorro y de inversión), sobre el ingreso según el tamaño de los parámetros.

De manera similar, la sencillez extrema del modelo es su defecto principal. No se incluye ninguna de las medicinas en contra del estancamiento como el uso extensivo del crédito y de la publicidad, el militarismo o los gastos sociales de corte anti-depresivo, aunque éstas mismas causan otros efectos en su contra (a favor del estancamiento) como es el caso de las altas tasas de interés que predominan en el mundo capitalista en la actualidad. Sin embargo, el objetivo de Sweezy no fue exponer un modelo completo del capitalismo sino de una de las tendencias principales del capitalismo maduro.

46 Si $f(k)=10[1-\exp (-\mathrm{k} / 10)] \mathrm{y}$ $\mathrm{g}(\mathrm{k})=[1-\exp (-10 \mathrm{k})] / 10$,

tenemos $\mathrm{f}(1) / \mathrm{I}(1) \stackrel{\text { (1) }}{=} 0.46385$ mientras que $\mathrm{f}(2) / \mathrm{I}(2)=0.62234$, etc. No creo que exista una función de consumo de esta clase que podía tener validez en el tiempo para cualquier economía.

47 Aunque las dos ideas fueron tratadas antes por Kahn en el caso del multiplicador y por Aftalion y J. M. Clark en el caso del acelerador.

48 Para un resumen del trabajo de estos autores, véase R. G. D. Allen, Macroeconomic Theory, Macmillan and Co., Ltd., London, 1967, capítulo 17. 


\section{8) La importancia del modelo para la periferia del capitalismo}

Recordamos la conclusión de Sweezy acerca de la propensión del capitalismo desarrollado (central) al estancamiento en cuyo caso será imposible cimentar en la periferia una política que dependa de las exportaciones para elevar su nivel productivo. Así, pues, será posible para algunos países periféricos, lograr un crecimiento rápido en base a exportaciones al Centro. De hecho durante los años cincuenta y sesenta, países como Corea del Sur, Taiwan, Brasil y en el caso de México, la franja fronteriza del Norte, han podido aumentar sus ingresos basándose en la importación de capital extranjero que exporta al Centro la producción de la Periferia. Es decir, el motor del modelo se ubica en el Centro. En estos casos, el estancamiento del Centro causa una crisis económica y financiera en la Periferia que amortigua la crisis del Centro y por lo tanto importa su estancamiento. Es claro que el estancamiento de la Periferia implica consecuencias desastrosas para el modelo planteado que es precisamente la situación actual de esta parte de la Periferia.

En el caso de México, aunque su principal producto de exportación goza de un "buen" precio y de una "buena" demanda, el estancamiento del Centro no garantiza una creciente demanda de petróleo, mientras que la dependencia mexicana de éste aumenta día tras día. En esta situación podemos concluir lo siguiente:

1) Ni siquiera el petróleo garantiza ingresos crecientes para México a pesar de los aumentos drásticos de los precios en los últimos años.

2) La política de exportaciones (camarones; fresas, zapatos, etc.) al Centro y de mayor vinculación con el Centro (el turismo, la mayor integración financiera, etc.) acarrearán a México constantes ajustes hacia menores ingresos y mayores egresos que los anticipados.

3) Una mayor desvinculación del Centro y una mayor atención interna serán indispensables para sostener un crecimiento elevado de la economía mexicana.

\section{Conclusiones}

El ingreso nacional tiene que crecer en forma acelerada para evitar momentáneamente el estancamiento. Estas tasas altísimas de crecimiento se obtienen solamente durante los grandes auges tales como 
los que suceden la apertura ("Opening-up" como si fueran latas de sardinas) de nuevos territorios (que permite el saqueo en escala y la consecuente elevación de $\mathrm{k}$ ), y "mercados" (la incorporación de grandes masas humanas al aparato acumulativo del capitalista) como los imperialismo europeos, norteamericano y japonés de los siglos XIII a XIX, ${ }^{49}$ algún invento de importancia extraordinaria ${ }^{50} \mathrm{o}$ algún auge especulativo ${ }^{51}$. Dejando de lado estas posibilidades, vemos que el mismo desarrollo de la economía capitalista produce su estancamiento.

Entonces, es justificado considerar, junto con Sweezy, que el estancamiento es una enfermedad crónica de la vejez del capitalismo. El Apéndice, como el resto del trabajo de Sweezy, es un paso más hacia el entendimiento de las enfermedades del monstruo dentro del cual vivimos. Esto no quiere decir que Sweezy no podría haberse equivocado o que el modelo es perfecto. Las dos posibilidades existen pero en verdad son irrelevantes, porque en última instancia lo que hace historia no son las derivadas sino las desigualdades.

\footnotetext{
49 Bleaney alega "un incremento constante de las tasas de crecimiento -a largo plazo-- en la historia de los paises capitalistas avanzados" en la p. 150 del libro ya citado. Tomando tasas de crecimiento por un período de tiempo largo (como un siglo) vemos tasas superiores para la mayoría de los países capitalistas desarrollados con algunas excepciones notables, como la Gran Bretaña que fue el primer país capitalista del siglo pasado. Es decir, el país con la historia más larga de los países capitalistas industriales avanzados, está dentro del rango de estancamiento de Sweezy (I $\leqslant 0$ ). Por otra parte, lo que es "largo plazo" es flexible. Por ejemplo, si tomamos el período $1945-1980$ vemos una tasa positiva y creciente de ingresos por aproximadamente dos terceras partes del período, pero una tasa decreciente e inclusive negativa de ingresos en la tercera parte del período.

50 Creo que Sweezy estaría de acuerdo en clasificar el automóvil en esta categoría hasta los años sesenta para la economía norteamericana.

51 Como el "South Sea Buble" en Inglaterra o 1929 en los EE.UU.
} 